\title{
RANDOM POWER SERIES GENERATED BY ERGODIC TRANSFORMATIONS
}

\author{
JUDY HALCHIN AND KARL PETERSEN
}

ABSTRACT. Generalizing classical studies of power series with sequences of independent random variables as coefficients, we study series of the forms

$$
g_{x, \phi}(z)=\sum_{n=0}^{\infty} \phi\left(T^{n} x\right) z^{n} \quad \text { and } f_{x, \phi}(z)=\sum_{n=1}^{\infty} \phi(x) \phi(T x) \cdots \phi\left(T^{n-1} x\right) z^{n} \text {, }
$$

where $T$ is an ergodic measure-preserving transformation on a probability space $(X, \mathscr{B}, \mu)$ and $\phi$ is a measurable complex-valued function which is a.e. nonzero.

When $f_{x . \phi}$ is entire, its order of growth at infinity measures the speed of divergence of the ergodic averages of $\log |\phi|$. We give examples to show that any order is possible for any $T$ and that different orders are possible for fixed $\phi$. For fixed $T$, the set of $\phi$ which produce infinite order is residual in the subset of $L^{1}(X)$ consisting of those $\phi$ which are a.e. nonzero and produce entire $f_{x, \phi}$. As in a theorem of Pólya for gap series, if $f_{x, \phi}$ is entire and has finite order, then it assumes every value infinitely many times.

The functions $\phi \in L^{1}(X)$ for which $g_{x, \phi}$ is rational a.e. are exactly the finite sums of eigenfunctions of $T$; their poles are all simple and are the inverses of the corresponding eigenvalues. By combining this result with a skew product construction, we can also characterize when $f_{x, \phi}$ is rational, provided that $\phi$ takes one of several particular forms.

1. Introduction. We are interested in studying power series with sequences of coefficients, $\left(a_{n}\right)$, generated by a measure-preserving transformation, $T$, in one of two ways: if $T$ is defined on a measure space, $(X, \mathscr{B}, \mu)$, and $\phi: X \rightarrow \mathbf{C}$ is a measurable function, then either $a_{n}=\phi \circ T^{n}$ or $a_{n}=\phi \cdot \phi \circ T \cdot \phi \circ T^{2} \cdots \phi \circ T^{n-1}$, $n=1,2, \ldots$.

R.E.A.C. Paley and A. Zygmund [7] studied the series

$$
\sum_{n=0}^{\infty} a_{n} c_{n} z^{n}
$$

where $\left(a_{n}\right)$ is the Rademacher sequence and $\left(c_{n}\right)$ is a fixed sequence of complex numbers. The Rademacher sequence,

$$
a_{n}= \begin{cases}1 & \text { on }\left[2 k / 2^{n},(2 k+1) / 2^{n}\right), \\ -1 & \text { on }\left[(2 k+1) / 2^{n},(2 k+2) / 2^{n}\right),\end{cases}
$$

Received by the editors May 30, 1985.

1980 Mathematics Subject Classification (1985 Revision). Primary 28D05, 30B20; Secondary 30D15.

Key words and phrases. Random power series, ergodic measure-preserving transformation, order of growth, lacunary series, rational function.

The research of the second author was supported in part by NSF grants MCS-8001590 and DMS8400730 . 
can be viewed as $a_{n}=\phi \circ T^{n}$, where $T$ is the Bernoulli shift $B\left(\frac{1}{2}, \frac{1}{2}\right)$ on the set $\{-1,1\}^{\mathbf{Z}}$ and $\phi$ is a coordinate function. H. Steinhaus $[10,11]$ considered the slightly more general case where $\left(a_{n}\right)$ is the Steinhaus sequence, $a_{n}=e^{2 \pi i \gamma_{n}}$, where $\left(\gamma_{n}\right)$ is a sequence of independent, uniformly distributed random variables. The properties of these series depend on the size of the coefficients $\left(c_{n}\right)$, and Paley, Zygmund and Steinhaus related properties such as inclusion in $L^{q}(\theta)$, continuity on the closed unit disk, and capability of analytic continuation outside the unit disk to summability properties of $\left(c_{n}\right)$. Some more recent results have related summability properties of $\left(c_{n}\right)$ to inclusion of the series in the spaces of Bloch functions and VMOA (see Anderson, Clunie, and Pommerenke [2] and Sledd [9]).

All of these results depend strongly on the independence of the coefficients, and most of them fail in the dependent case. The use of an ergodic transformation in forming the coefficients better allows analysis of the dependent case, in addition to providing the opportunity to study ergodic-theoretic properties of the underlying transformation by means of studying the power series. Specifically, the analytic properties of the power series

$$
g_{x, \phi . T}(z)=\sum_{n=0}^{\infty} \phi\left(T^{n} x\right) z^{n}
$$

and

$$
f_{x, \phi, T}(z)=\sum_{n=0}^{\infty} \phi(x) \phi(T x) \cdots \phi\left(T^{n-1} x\right) z^{n}
$$

can sometimes be directly related to ergodic-theoretic properties of $T$. Ionescu Tulcea [4] established several such relationships for the series $g_{x, \phi, T}$, connecting the radius of convergence to the ergodicity of $T$ and the existence of radial limits of $g_{x, \phi, T}$ to the weak mixing of $T$. She also showed that the singular points of $g_{x, \phi, T}$ on the unit circle must be in the spectrum of $U_{T}$ (the unitary operator on $L^{2}(X)$ induced by $T$ ) (see Ionescu Tulcea [5]).

In $\S 2$, we study the order of growth of the random power series $f_{x, \phi, T}$ when it represents an entire function, and the relationship of its order to the rate of divergence of the ergodic averages of the function $\log |\phi|$. (See Aaronson [1] for another discussion of rates of divergence.) Examples are given showing that, for a given transformation $T$, any order is possible. We then show that infinite order is most typical, in the sense that, among the functions $\phi$ for which the power series represents an entire function, those giving infinite order form a residual set in the $L^{1}(X)$ topology.

In $\S 3$ we discuss the similarity between gap series and the random series $f_{x, \phi, T}$ when it represents an entire function. A theorem of Pólya states that an entire, transcendental gap series of finite order assumes every value infinitely many times. We prove the same theorem, replacing only the gap series with the random series.

$\$ 4$ studies conditions under which a random power series represents a rational function. It is shown that the series $g_{x, \phi, T}$ represents a rational function for $\phi$ in $L^{1}(X)$ if and only if $\phi$ is a finite sum of eigenfunctions of $T$, and, in this case, the 
poles of the rational function are all simple and occur at the inverses of the corresponding eigenvalues. For several special classes of the function $\phi$ we characterize those power series of the form $f_{x, \phi, T}$ which represent rational functions, applying the theorem for the series $g_{x, \phi, T}$ in two of these cases.

We will use the following notation throughout. $T$ will be an invertible, ergodic, measure-preserving transformation on a nonatomic probability space $(X, \mathscr{B}, \mu) . \boldsymbol{\phi}$ will be a measurable, complex-valued function on $X$. When working with the series $f_{x, \phi, T}$, we will always assume that $\phi(x) \neq 0$ holds almost everywhere to ensure that $f_{x, \phi, T}$ is not a polynomial. More details for some of the arguments can be found in the Ph.D. dissertation of the first author (see Halchin [6]).

2. Possible and prevalent orders of growth. We examine first the series

$$
f_{x, \phi, T}(z)=\sum_{n=0}^{\infty} \phi(x) \phi(T x) \cdots \phi\left(T^{n-1} x\right) z^{n} .
$$

Its radius of convergence is given by

$$
\begin{aligned}
r & =\left[\limsup _{n \rightarrow \infty}\left|\phi(x) \phi(T x) \cdots \phi\left(T^{n-1} x\right)\right|^{1 / n}\right]^{-1} \\
& =\exp \left(-\limsup _{n \rightarrow \infty} \log \left|\phi(x) \phi(T x) \cdots \phi\left(T^{n-1} x\right)\right|^{1 / n}\right) \\
& =\exp \left(-\limsup _{n \rightarrow \infty} \frac{1}{n} \sum_{j=0}^{n-1} \log \left|\phi\left(T^{j} x\right)\right|\right) \\
& =\exp \left(-\int_{X} \log |\phi| d \mu\right) .
\end{aligned}
$$

We first consider the case where $\int_{X} \log |\phi| d \mu=-\infty$ (that is, $\int_{X} \log ^{+}|\phi| d \mu<\infty$ and $\left.\int_{X} \log ^{-}|\phi| d \mu=\infty\right)$, making $f_{x, \phi, T}$ an entire function. The order of growth of an entire function, $f$, is defined as

$$
\rho=\inf \left\{A: \max _{|z|=r}|f(z)| \leqslant e^{r^{A}} \text { for all } r \text { sufficiently large }\right\} .
$$

If $f$ has power series $f(z)=\sum_{n=0}^{\infty} a_{n} z^{n}$, then $\rho$ is also given by

$$
-\frac{1}{\rho}=\limsup _{n \rightarrow \infty} \frac{\log \left|a_{n}\right|}{n \log n} .
$$

In the case of $f_{x, \phi, T}$ this becomes

$$
\begin{aligned}
-\frac{1}{\rho} & =\limsup _{n \rightarrow \infty} \frac{\log \left|\phi(x) \cdots \phi\left(T^{n-1} x\right)\right|}{n \log n} \\
& =\limsup _{n \rightarrow \infty} \frac{\frac{1}{n} \sum_{j=0}^{n-1} \log \left|\phi\left(T^{j} x\right)\right|}{\log n} .
\end{aligned}
$$


Thus the order of $f_{x, \phi, T}$ is a measurement of the speed of divergence to $-\infty$ of the ergodic averages of $\log |\phi|$ as compared to $\log n$. For any given $T, f_{x, \phi, T}$ can attain any order, $0 \leqslant \rho \leqslant \infty$, as the following examples show.

EXAMPLE 1. $f_{x, \phi . T}$ having order 0 . Fix any partition, $\left\{A_{1}, A_{2}, A_{3}, \ldots\right\}$, of $X$ such that $\mu\left(A_{i}\right)>0$ for every $i$. We define

$$
\phi=e^{-\sum_{i=1}^{\infty} a_{i} \chi_{A_{i}}}, \quad a_{i}=\frac{2 n_{i} \log n_{i+1}}{\mu\left(A_{i}\right)},
$$

with the sequence $\left(n_{i}\right)$ defined as follows. Using Egoroff's Theorem we can find a measurable set $B \subset X$ such that $\mu(B)>0$ and such that

$$
\frac{1}{n} \sum_{j=0}^{n-1} \chi_{A_{i}}\left(T^{j} x\right) \rightarrow \mu\left(A_{i}\right)
$$

uniformly on $B$ for every $i$. We choose $\left(n_{i}\right)$ to be an increasing sequence of integers such that, for each $i$,

$$
\left|\frac{1}{n} \sum_{j=0}^{n-1} \chi_{A_{i}}\left(T^{j} x\right)-\mu\left(A_{i}\right)\right|<\frac{\mu\left(A_{i}\right)}{2}
$$

for every $n \geqslant n_{i}$ and every $x$ in $B$.

We now see that

$$
\begin{aligned}
\int_{X} \log |\phi| d \mu & =\int_{X}-\sum_{i=1}^{\infty} a_{i} \chi_{A_{i}} d \mu=-\sum_{i=1}^{\infty} a_{i} \mu\left(A_{i}\right) \\
& =-\sum_{i=1}^{\infty} 2 n_{i} \log n_{i+1}=-\infty
\end{aligned}
$$

and we see that the order of $f_{x, \phi, T}$ is zero as follows. For any $n$ we define $k(n)$ by $n_{k(n)} \leqslant n<n_{k(n)+1}$. For every $x$ in $B$ we have

$$
\begin{aligned}
-\frac{1}{\rho} & =\limsup _{n \rightarrow \infty} \frac{-1}{\log n} \sum_{i=0}^{\infty}\left[\frac{1}{n} \sum_{j=0}^{n-1} \chi_{A_{i}}\left(T^{j} x\right)\right] a_{i} \\
& \leqslant \limsup _{n \rightarrow \infty} \frac{-1}{\log n}\left[\frac{1}{n} \sum_{j=0}^{n-1} \chi_{A_{k(n)}}\left(T^{j} x\right)\right] a_{k(n)} \\
& \leqslant \limsup _{n \rightarrow \infty} \frac{-1}{\log n}\left[\mu\left(A_{k(n)}\right)-\frac{\mu\left(A_{k(n)}\right)}{2}\right] a_{k(n)} \\
& =\limsup _{n \rightarrow \infty} \frac{-\mu\left(A_{k(n)}\right)}{2 \log n} \frac{2 n_{k(n)} \log n_{k(n)+1}}{\mu\left(A_{k(n)}\right)}=-\infty .
\end{aligned}
$$

In the following example we use a sequence, $\left(n_{k}\right)$, in a similar manner. Here, we hold $n_{k}$ constant until it is smaller than $\sqrt{\log k}$, then allow it to jump to a very high value where it will remain until $n_{k}<\sqrt{\log k}$ again, as pictured in Figure 1. Thus we have a subsequence where the $n$th ergodic average is small compared to $\log n$, but the values at the jumps are large enough to make $\log ^{-}|\phi|$ nonintegrable. 


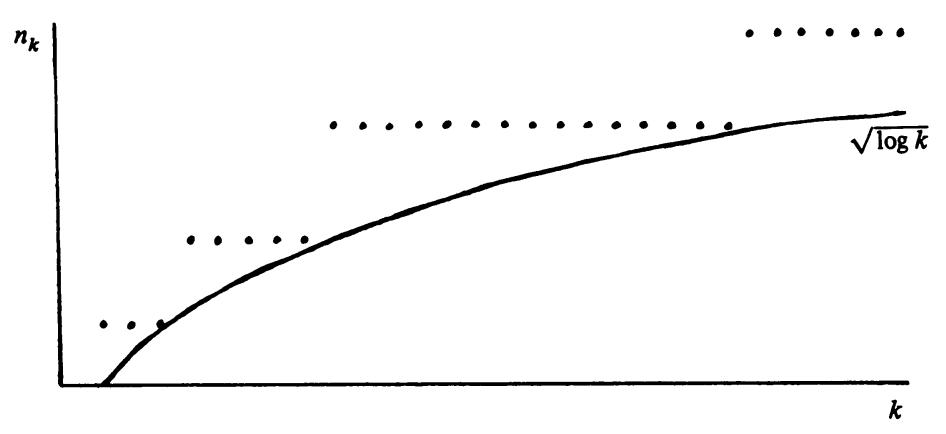

FIGURE 1

EXAmple 2. $f_{x, \phi, T}$ having order $\infty$. Choose any partition $\left\{A_{1}, A_{2}, A_{3}, \ldots\right\}$ of $X$ with $\mu\left(A_{n}\right)>0$ for every $n$, and an increasing sequence, $\left(n_{k}\right)$, of positive integers such that $n_{1}=1$ and

$$
\sum_{k=1}^{\infty}(k+1) \mu\left(\bigcup_{n=n_{k}+1}^{\infty} A_{n}\right)<\infty .
$$

The function $\phi$ will have the constant value $e^{-a_{k}}$ on $A_{n_{k}} \cup \cdots \cup A_{n_{k+1}-1}$ for each $k$, where $a_{1}=1 / \mu\left(A_{1}\right)$ and

$$
a_{k}= \begin{cases}a_{k-1} & \text { if } a_{k-1} \geqslant \sqrt{\log k}, \\ \max \left\{a_{k-1}, 1 / \mu\left(A_{n_{k}}\right)\right\} & \text { if } a_{k-1}<\sqrt{\log k}\end{cases}
$$

for $k>1$. Note that $\left(a_{k}\right)$ is nondecreasing and all $a_{k}>0$. Furthermore, there is a subsequence, $\left(a_{k_{i}}\right)$, with

$$
a_{k_{i}-1}<\sqrt{\log k_{i}} \text { and } a_{k_{i}} \geqslant \frac{1}{\mu\left(A_{n_{k_{i}}}\right)}
$$

for every $i$. Therefore

Now consider the set

$$
\begin{aligned}
\int_{X} \log \mid \phi \cdot d \mu & =\int_{X}\left(-\sum_{k=1}^{\infty} a_{k} \chi_{A_{n_{k}} \cup \cdots \cup A_{n_{k+1}}-1}\right) d \mu \\
& \leqslant-\sum_{i=1}^{\infty} a_{k_{i}} \sum_{n=n_{k_{i}}+1}^{n_{k_{i}}-1} \mu\left(A_{n}\right) \\
& \leqslant-\sum_{i=1}^{\infty} a_{k_{i}} \mu\left(A_{n_{k_{i}}}\right) \leqslant-\sum_{i=1}^{\infty} 1=-\infty
\end{aligned}
$$

$$
B=\bigcup_{K=1}^{\infty} \bigcap_{k=K}^{\infty} B_{k}=\bigcup_{K=1}^{\infty} \bigcap_{k=K}^{\infty} \bigcap_{j=0}^{k} T^{-j}\left(A_{1} \cup \cdots \cup A_{n_{k}}\right) .
$$

This is the set of all $x$ whose first $k+1$ iterates stay in $A_{1} \cup \cdots \cup A_{n_{k}}$ for all $k$ sufficiently large. Condition (1) together with the Borel-Cantelli Lemma implies that $\mu(B)=1$. 


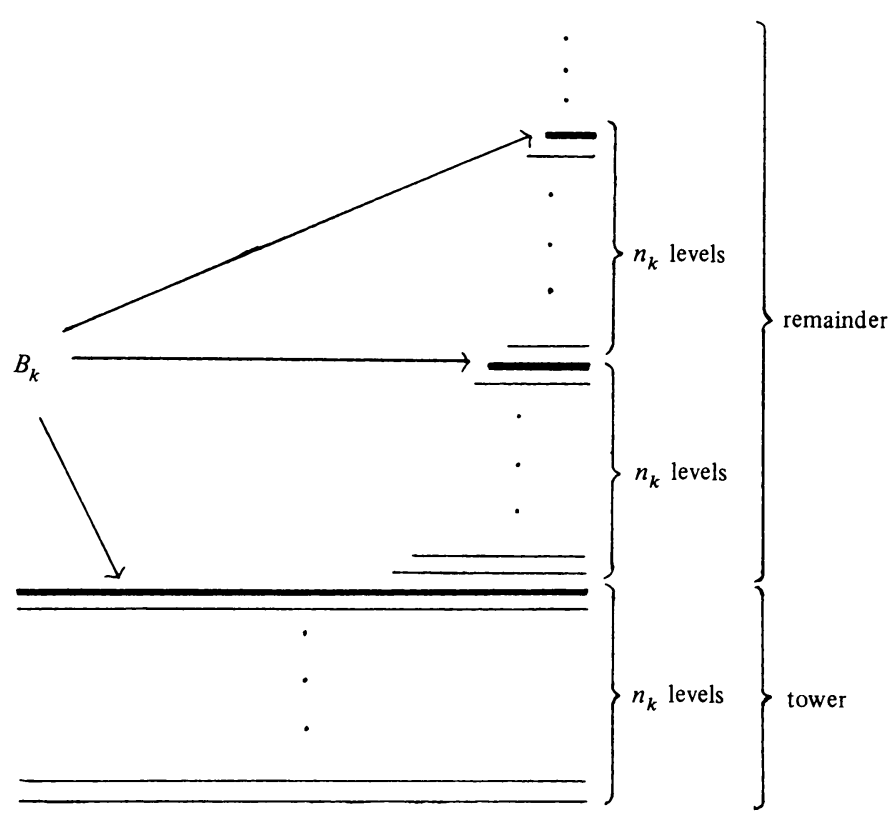

Figure 2

Now fix any $x$ in $B$. Whenever $i$ is large enough, we have $x, T x, \ldots, T^{k_{1}-1} x$ all in $A_{1} \cup \cdots \cup A_{n_{h_{1}-1}}$, so

$$
\begin{aligned}
\frac{\sum_{j=0}^{k_{1}-1} \log \left|\phi\left(T^{j} x\right)\right|}{k_{i} \log k_{i}} & =\frac{\sum_{j=0}^{k_{i}-1} \sum_{m=1}^{\infty} a_{m} \chi_{A_{n_{m}}} \cup \cdots \cup A_{n_{m+1}-1}\left(T^{j} x\right)}{k_{i} \log k_{i}} \\
& =\frac{-\sum_{j=0}^{k_{i}-1} \sum_{m=1}^{k_{1}-1} a_{m} \chi_{A_{n_{m}}} \cup \cdots \cup A_{n_{m+1}-1}\left(T^{j} x\right)}{k_{i} \log k_{i}} \\
& \geqslant \frac{-\sum_{j=0}^{k_{1}-1} a_{k_{1}-1}}{k_{i} \log k_{i}}=\frac{-k_{i} a_{k_{i}-1}}{k_{i} \log k_{i}}>\frac{-\sqrt{\log k_{i}}}{\log k_{i}}
\end{aligned}
$$

which has limit 0 , so $\rho=\infty$.

EXAMPLE 3. $f_{x, \phi, T}$ having finite, nonzero order $\alpha$. We will use a sequence, $\left(n_{k}\right)$, of positive integers and a decreasing sequence, $\left(\varepsilon_{k}\right)$, of positive numbers to choose a tower of height $n_{k}$ with remainder of measure less than $\varepsilon_{k}$ for each $k=1,2,3, \ldots$ We define $B_{k}$ to be the set consisting of the top level of the tower and every $n_{k}$ th level above that, as pictured in Figure 2. Now $\phi$ will be of the form

$$
\phi=e^{-\sum_{k=1}^{\infty} f_{k}},
$$

where $f_{k}$ is nonzero only on $B_{k}$. The sequence $\left(n_{k}\right)$ will be defined by $n_{1}=2$ and $n_{k}=\left[\left(n_{1} n_{2} \cdots n_{k-1}\right)^{1+1 / k}\right]$ for $k \geqslant 2$, where [ ] denotes the greatest integer function. We define a closely related sequence, $\left(n_{k}^{\prime}\right)$, by

$$
n_{1}^{\prime}=2 \text { and } n_{k}^{\prime}=\left[\left(n_{1} n_{2} \cdots n_{k}\right)^{1+1 / 2^{k+1}}\right] \text { for } k \geqslant 2 .
$$

We see easily that $n_{k} \leqslant n_{k}^{\prime} \leqslant n_{k+1}$. 
Let $D$ be the set of points, $x$, such that for sufficiently large $i$ none of the first $n_{i}^{\prime}$ points of the orbit of $x$ are contained in any $B_{k}$ for $k>i$. Thus, for any $x$ in $D$, if $i$ is large enough, then

$$
\sum_{k=1}^{\infty} f_{k}\left(T^{j} x\right)=\sum_{k=1}^{i} f_{k}\left(T^{j} x\right)
$$

for every $j=0,1, \ldots, n_{i}-1$. Using $\mu\left(B_{k}\right) \leqslant 1 / n_{k}$, we can write

$$
\begin{aligned}
\mu\left(D^{c}\right) & =\mu\left[\bigcap_{I=1}^{\infty} \bigcup_{i=I}^{\infty} \bigcup_{k=i+1}^{\infty} \bigcup_{j=0}^{n_{i}^{\prime}-1} T^{-j}\left(B_{k}\right)\right] \\
& \leqslant \lim _{I \rightarrow \infty} \sum_{i=I}^{\infty} \sum_{k=i+1}^{\infty} \mu\left[\bigcup_{j=0}^{n_{i}^{\prime}-1} T^{-j}\left(B_{k}\right)\right] \\
& \leqslant \lim _{I \rightarrow \infty} \sum_{i=I}^{\infty} \sum_{k=i+1}^{\infty} n_{i}^{\prime} \mu\left(B_{k}\right) \\
& \leqslant \lim _{I \rightarrow \infty} \sum_{i=I}^{\infty} n_{i}^{\prime} \sum_{k=i+1}^{\infty} \frac{1}{n_{k}} .
\end{aligned}
$$

An easy induction argument shows that $n_{k} \geqslant 2^{2^{k-2}}$ for every $k$. Using this together with the definitions of $n_{i}$ and $n_{i}^{\prime}$, one can readily check that

$$
\sum_{i=1}^{\infty} n_{i}^{\prime} \sum_{k=i+1}^{\infty} \frac{1}{n_{k}}<\infty
$$

so we must have $\mu(D)=1$.

Let $C$ be the set of points, $x$, such that for sufficiently large $k$ the first $n_{k+1}$ points of the orbit of $x$ lie in the tower of height $n_{k}$. Thus, for any $x$ in $C$, if $k$ is large enough, then $x, T x, \ldots, T^{n_{k+1}-1} x$ hits $B_{k}$ exactly once every $n_{k}$ steps. It follows from writing

$$
\text { . } C=\bigcup_{K=1}^{\infty} \bigcap_{k=K}^{\infty} \bigcup_{j=0}^{n_{k+1}-1} T^{-j}\left(C_{k}\right)
$$

(where $C_{k}$ is the tower of height $n_{k}$ ) and using the Borel-Cantelli Lemma that $\mu(C)=1$ if $\varepsilon_{k}=1 / 2^{k} n_{k+1}$ for every $k$.

Using $n_{i} \geqslant 2^{2^{i-2}}$ for every $i, 2^{i-2} i /(i+1)(i+1)>4$ for $i \geqslant 8$, and the definition of $n_{i}$, we get $n_{i+1}>16 n_{i}^{2}$ for all $i \geqslant 8$. The following limit identities follow easily from this and from the definitions of $\left(n_{i}\right)$ and $\left(n_{i}^{\prime}\right)$.

$$
\begin{gathered}
\lim _{i \rightarrow \infty} \frac{n_{i}}{n_{i}^{\prime}}=\lim _{i \rightarrow \infty} \frac{n_{i}}{n_{i+1}}=0, \quad \lim _{i \rightarrow \infty} \frac{\log n_{i}}{\log n_{i+1}}=\frac{1}{2}, \\
\lim _{i \rightarrow \infty} \frac{\log \left(n_{1} n_{2} \cdots n_{i-1}\right)}{\log n_{i}}=\lim _{i \rightarrow \infty} \frac{\log \left(n_{1} n_{2} \cdots n_{i}\right)}{\log n_{i}^{\prime}}=1 .
\end{gathered}
$$

We now partition $B_{k}$ into $B_{k, 0} \cup B_{k, 1} \cup \cdots \cup B_{k, n_{k}-1}$ by defining $B_{k, i}$ to be the set of points in $B_{k}$ whose backward orbit first hits $B_{k}$ again after exactly $n_{k}+i$ 
steps, and we define

$$
f_{k}=\frac{1}{\alpha} \log n_{k} \sum_{i=0}^{n_{k}-1}\left(n_{k}+i\right) \chi_{B_{k, i}} .
$$

We consider the sum $\sum_{j=0}^{n-1} f_{k}\left(T^{j} x\right)$ by starting at a point, $x$, in $T B_{k}$ and traveling along its forward orbit. Each time we get a contribution to the sum it will be $\frac{1}{\alpha} \log n_{k}$ times the number of steps taken. Thus, we must have

$$
\sum_{j=0}^{n-1} f_{k}\left(T^{j} x\right)=\frac{1}{\alpha} n \log n_{k}
$$

if we started in $T B_{k}$ and ended at $B_{k}$. However, if we do not start at a point in $T B_{k}$ or do not end exactly at $B_{k}$, then we have

$$
\sum_{j=0}^{n-1} f_{k}\left(T^{j} x\right)=\frac{1}{\alpha}(n+\operatorname{error}(x, n, k)) \log n_{k},
$$

where $-\left(2 n_{k}-2\right) \leqslant \operatorname{error}(x, n, k) \leqslant 2 n_{k}-2$.

At this point it can easily be checked that $\int_{X} \log |\phi| d \mu=-\infty$, as desired.

Now fix any $x$ in $C \cap D$. Let $n$ be large enough that the defining properties of both $C$ and $D$ hold for $x$. We define $i$ by $n_{i} \leqslant n<n_{i+1}$. Then

$$
\begin{aligned}
-\frac{1}{\rho} & =\limsup _{n \rightarrow \infty} \frac{-1}{n \log n} \sum_{j=0}^{n-1} \sum_{k=1}^{\infty} f_{k}\left(T^{j} x\right) \\
& \leqslant \limsup _{n \rightarrow \infty} \frac{-1}{n \log n} \sum_{k=1}^{i} \sum_{j=0}^{n-1} f_{k}\left(T^{j} x\right) \\
& =\limsup _{n \rightarrow \infty} \frac{-1}{n \log n} \sum_{k=1}^{i} \frac{1}{\alpha}(n+\operatorname{error}(x, n, k)) \log n_{k} .
\end{aligned}
$$

We write $n=m n_{i}+p$, where $0 \leqslant p \leqslant n_{i}-1$ and $1 \leqslant m \leqslant\left(n_{i+1}-1\right) / n_{i}$. Since $x$ is in $C$ we have error $\left(x, m n_{i}+p, i\right) \geqslant-p$, so

$$
\begin{aligned}
\frac{n+\operatorname{error}(x, n, i)}{n \log n} & =\frac{\left(m n_{i}+p\right)+\operatorname{error}\left(x, m n_{i}+p, i\right)}{\left(m n_{i}+p\right) \log \left(m n_{i}+p\right)} \\
& \geqslant \frac{m n_{i}}{\left(m n_{i}+p\right) \log \left(m n_{i}+p\right)} .
\end{aligned}
$$

This function of $m$ and $p$ attains its minimum on the given domain at $m=$ $\left(n_{i+1}-1\right) / n_{i}$ and $p=n_{i}-1$. Now we have

$$
\frac{n+\operatorname{error}(x, n, i)}{n \log n} \geqslant \frac{n_{i+1}-1}{\left(n_{i+1}+n_{i}-2\right) \log \left(n_{i+1}+n_{i}-2\right)} .
$$


Therefore,

$$
\begin{aligned}
& -\frac{1}{\rho} \leqslant \limsup _{n \rightarrow \infty} \frac{-1}{\alpha}\left[\frac{(n+\operatorname{error}(x, n, i)) \log n_{i}}{\log n}+\frac{\sum_{k=1}^{i-1}(n+\operatorname{error}(x, n, k)) \log n_{k}}{n \log n}\right] \\
& \leqslant \limsup _{n \rightarrow \infty} \frac{-1}{\alpha}\left[\frac{\left(n_{i+1}-1\right) \log n_{i}}{\left(n_{i+1}+n_{i}-2\right) \log \left(n_{i+1}+n_{i}-2\right)}+\frac{\sum_{k=1}^{i-1}\left(n-2 n_{k}\right) \log n_{k}}{n \log n}\right] \\
& \leqslant \limsup _{n \rightarrow \infty} \frac{-1}{\alpha} \frac{n_{i+1}-1}{n_{i+1}+n_{i}-2} \cdot \frac{\log n_{i}}{\log \left(n_{i+1}+n_{i}-2\right)} \\
& +\limsup _{n \rightarrow \infty} \frac{-1}{\alpha} \frac{\sum_{k=1}^{i-1} \log n_{k}}{\log n}+\limsup _{n \rightarrow \infty} \frac{2}{\alpha} \frac{\sum_{k=1}^{i-1} n_{k} \log n_{k}}{n \log n} \\
& \leqslant \frac{-1}{\alpha} \frac{1}{\liminf _{i \rightarrow \infty}\left[n_{i+1} /\left(n_{i+1}-1\right)+\left(n_{i}-2\right) /\left(n_{i+1}-1\right)\right]} \lim _{i \rightarrow \infty} \frac{\log n_{i}}{\log n_{i+1}+\log 2} \\
& +\limsup _{i \rightarrow \infty} \frac{-1}{\alpha} \frac{\log \left(n_{1} \cdots n_{i-1}\right)}{\log n_{i+1}}+\limsup _{i \rightarrow \infty} \frac{2}{\alpha} \frac{n_{i-1}}{n_{i}} \frac{\log \left(n_{1} \cdots n_{i-1}\right)}{\log n_{i}} \\
& =\frac{-1}{\alpha} \cdot \frac{1}{1+0} \cdot \frac{1}{2}+\frac{-1}{\alpha} \cdot \frac{1}{2}+\frac{2}{\alpha} \cdot 0 \cdot 1=-\frac{1}{\alpha} \text {. }
\end{aligned}
$$

Thus $\rho \leqslant \alpha$.

Now looking at the subsequence where $n=n_{i}^{\prime}$ and using the fact that $x$ is in $D$, we have

$$
\begin{aligned}
\frac{1}{\rho} & =\limsup _{i \rightarrow \infty} \frac{-1}{n_{i}^{\prime} \log n_{i}^{\prime}} \sum_{j=0}^{n_{i}^{\prime}-1} \sum_{k=1}^{i} f_{k}\left(T^{j} x\right) \\
& =\limsup _{i \rightarrow \infty} \frac{-1}{n_{i}^{\prime} \log n_{i}^{\prime}} \sum_{k=1}^{i} \frac{1}{\alpha}\left(n_{i}^{\prime}+\operatorname{error}\left(x, n_{i}^{\prime}, k\right)\right) \log n_{k} \\
& \geqslant \limsup _{i \rightarrow \infty} \frac{-1}{\alpha} \frac{\sum_{k=1}^{i}\left(n_{i}^{\prime}+2 n_{k}\right) \log n_{k}}{n_{i}^{\prime} \log n_{i}^{\prime}} \\
& =\limsup _{i \rightarrow \infty} \frac{-1}{\alpha}\left[\frac{\log \left(n_{1} \cdots n_{i}\right)}{\log n_{i}^{\prime}}+2 \frac{n_{i}}{n_{i}^{\prime}} \frac{\log \left(n_{1} \cdots n_{i}\right)}{\log n_{i}^{\prime}}\right] \\
& =-\frac{1}{\alpha}[1+2 \cdot 0 \cdot 1]=-\frac{1}{\alpha} .
\end{aligned}
$$

Therefore, $p=\alpha$.

That the order depends on $T$ as well as $\phi$ is shown by the following example.

EXAMPLE 4. $f_{x, \phi, T}$ and $f_{x, \phi, S}$ with different orders. Let $X=[0,1)$ and let $\left\{A_{i}\right\}$ be the partition $A_{1}=\left[0, \frac{1}{2}\right), A_{2}=\left[\frac{1}{2}, \frac{3}{4}\right), A_{3}=\left[\frac{3}{4}, \frac{7}{8}\right)$, and so forth. We define

$$
\phi=e^{-\sum_{i=1}^{\infty} 2^{2} x_{A_{i}}}
$$

Clearly, $\int_{X} \log |\phi| d \mu=-\infty$.

We will define $T=\lim _{k \rightarrow \infty} T_{k}$ and $S=\lim _{k \rightarrow \infty} S_{k}$ with cutting and stacking procedures designed so that an orbit under $T$ hits only small values of $\log |\phi|$ for a very long time, while an orbit under $S$ must hit fairly large values of $\log |\phi|$ regularly. 
We first cut $A_{1}$ into two equal intervals and stack them, then put $A_{2}$ on top. We next cut this stack into eight pieces and stack them, then put $A_{3}$, cut into four pieces, on top. At the $k$ th step we cut the previous stack into $2 k$ pieces and cut $A_{k+1}$ into $k$ ! pieces to go on top. $T_{k}$ is the transformation that moves points upward in the stack and is the identity on the top level of the stack and on $A_{k+2} \cup A_{k+3}$ $\cup \cdots$. At step $k$ we define $B_{k}$ to be the bottom $\left(1-1 / 2^{k}\right)$ th part of that portion of the $k$ th stack obtained by cutting up the old stack. $B_{k}$ will have height $2\left(2^{k}-1\right) k !\left(1-1 / 2^{k}\right)$. Furthermore, if we start in $B_{k}$ and travel $\left(\left(2^{k}-1\right) / 2^{k-1}\right) k$ ! steps, we will stay in $A_{1} \cup \cdots \cup A_{k}$ and will land in $A_{i}$ exactly $\left(2^{k-i} / 2^{k-1}\right) k$ ! times for $i=1,2, \ldots, k$. Since, for each $k$, the fraction of $B_{1}$ $\cap \cdots \cap B_{k-1}$ that is contained in $B_{k}$ is $1-1 / 2^{k}$, we see that

$$
\mu(B)=\mu\left(\bigcup_{k=1}^{\infty} B_{k}\right)=\frac{1}{2} \prod_{k=1}^{\infty}\left(1-\frac{1}{2^{k}}\right)>0
$$

Now choose $x$ in $B$. Let $k=k(n)$ be defined by

$$
\frac{2^{k-1}-1}{2^{k-2}}(k-1) !<n \leqslant \frac{2^{k}-1}{2^{k-1}} k !
$$

we have

$$
\begin{aligned}
-\frac{1}{\rho}= & \limsup _{n \rightarrow \infty} \frac{1}{n \log n} \sum_{j=0}^{n-1}\left(-\sum_{i=1}^{\infty} 2^{i} \chi_{A_{i}}\left(T^{j} x\right)\right) \\
& \geqslant \limsup _{k \rightarrow \infty} \frac{1}{\left(\left(2^{k}-1\right) / 2^{k-1}\right) k ! \log \left[\left(\left(2^{k}-1\right) / 2^{k-1}\right) k !\right]} \\
& \quad \sum_{j=0}^{\left(\left(2^{k}-1\right) / 2^{k-1}\right) k !-1}\left(-\sum_{i=1}^{\infty} 2^{i} \chi_{A_{i}}\left(T^{j} x\right)\right) \\
= & \limsup _{k \rightarrow \infty} \frac{1}{\left(\left(2^{k}-1\right) / 2^{k-1}\right) k ! \log \left[\left(\left(2^{k}-1\right) / 2^{k-1}\right) k !\right]} \sum_{i=1}^{k} 2^{i} \frac{2^{k-i}}{2^{k-1}} k ! \\
= & \limsup _{k \rightarrow \infty} \frac{-2 k ! k}{\left(\left(2^{k}-1\right) / 2^{k-1}\right) k !\left[\log \left(\left(2^{k}-1\right) / 2^{k-1}\right)+\log k !\right]}=0 .
\end{aligned}
$$

Thus, $f_{x, \phi, T}$ has order $\infty$ for almost every $x$.

$S$ is the usual von Neumann-Kakutani adding machine: we first stack $\left[\frac{1}{2}, 1\right)=A_{2}$ $\cup A_{3} \cup \cdots$ on top of $\left[0, \frac{1}{2}\right)=A_{1}$. Continuing, at the $k$ th step we cut the present stack in half and put the right-hand half on top. The new top level will be precisely $A_{k+1} \cup A_{k+2} \cup \cdots . S_{k}$ moves points in $A_{1} \cup \cdots \cup A_{k}$ up one level and leaves $A_{k+1} \cup A_{k+2} \cup \cdots$ fixed. Notice that every orbit hits $A_{k}$ exactly once every $2^{k}$ steps for every $k$. Now pick any $x$ and any $n$. Define $k=k(n)$ by $2^{k} \leqslant n<2^{k+1}$. 
We have

$$
\begin{aligned}
-\frac{1}{\rho} & =\limsup _{n \rightarrow \infty} \frac{1}{n \log n} \sum_{j=0}^{n-1} \log \left|\phi\left(S^{j} x\right)\right| \\
& =\limsup _{n \rightarrow \infty} \frac{1}{n \log n} \sum_{i=1}^{\infty}-2^{i} \sum_{j=0}^{n-1} \chi_{A_{i}}\left(S^{j} x\right) \\
& \leqslant \limsup _{k \rightarrow \infty} \frac{-1}{2^{k+1} \log 2^{k+1}} \sum_{i=1}^{k} 2^{i} \sum_{j=0}^{2^{k}-1} \chi_{A_{i}}\left(S^{j} x\right) \\
& =\limsup _{k \rightarrow \infty} \frac{-1}{2^{k+1}(k+1) \log 2} \sum_{i=1}^{k} 2^{i} \cdot \frac{2^{k}}{2^{i}} \\
& \leqslant \limsup _{k \rightarrow \infty} \frac{-k 2^{k}}{2^{k+1}(k+1) \log 2}=\frac{-1}{2 \log 2},
\end{aligned}
$$

so $\rho \leqslant 1 / 2 \log 2$.

We turn now to the question of which order is most typical. Let $\mathscr{M}$ be the spae of C-valued measurable functions on $X$ with the topology of convergence in measure. The following proposition states that, for the typical function $\phi$ in $\mathscr{M}$, the lim sup in measure of $(1 / n) \sum_{j=0}^{n-1} \log \left|\phi \circ T^{j}\right|$ is $\infty$ and the lim inf is $-\infty$.

Proposition. The set of functions $\phi$ in $\mathscr{M}$ for which the following property holds is second category in $\mathscr{M}$ : There exist sequences $\left(n_{i}\right)$ and $\left(n_{k}\right)$ such that $\left(1 / n_{i}\right) \sum_{j=0}^{n_{i}-1} \log \left|\phi \circ T^{j}\right| \rightarrow \infty$ in measure and $\left(1 / n_{k}\right) \sum_{j=0}^{n_{k}-1} \log \left|\phi \circ T^{j}\right| \rightarrow-\infty$ in measure.

Proof. We write the set described as

$$
\begin{aligned}
& {\left[\bigcap_{m=1}^{\infty} \bigcap_{N=1}^{\infty} \bigcup_{n=N}^{\infty}\left\{\phi \in \mathscr{M}: \mu\left(\left\{x: \frac{1}{n} \sum_{j=0}^{n-1} \log \left|\phi\left(T^{j} x\right)\right| \leqslant m\right\}\right)<\frac{1}{m}\right\}\right] } \\
& \cap\left[\bigcap_{m=1}^{\infty} \bigcap_{N=1}^{\infty} \bigcup_{n=N}^{\infty}\left\{\phi \in \mathscr{M}: \mu\left(\left\{x: \frac{1}{n} \sum_{j=0}^{n-1} \log \left|\phi\left(T^{j} x\right)\right| \geqslant-m\right\}\right)<\frac{1}{m}\right\}\right] \\
&= {\left[\bigcap_{m=1}^{\infty} \bigcap_{N=1}^{\infty} E_{m, n}\right] \cap\left[\bigcap_{m=1}^{\infty} \bigcap_{N=1}^{\infty} F_{m, N}\right] . }
\end{aligned}
$$

It is easily checked that each $E_{m, N}$ and each $F_{m, N}$ is dense in $\mathscr{M}$. To see that $E_{m, N}$ (the argument for $F_{m, N}$ is analogous) is open, consider

$$
E_{m, N}^{c}=\bigcap_{n=N}^{\infty}\left\{\phi \in \mathscr{M}: \mu\left(\left\{x: \frac{1}{n} \sum_{j=0}^{n-1} \log \left|\phi\left(T^{j} x\right)\right| \leqslant m\right\}\right) \geqslant \frac{1}{m}\right\} .
$$


Suppose that $\phi^{i} \rightarrow \phi$ in measure and $\phi^{i}$ is in $E_{m, n}^{c}$ for each $i$. Fix any $n \geqslant N$. Since, for any $\delta>0$,

$$
\begin{aligned}
\mu(\{x: & \left.\left.\frac{1}{n} \sum_{j=0}^{n-1} \log \left|\phi\left(T^{j} x\right)\right| \leqslant m+\delta\right\}\right) \\
\geqslant & \mu\left(\left\{x: \frac{1}{n} \sum_{j=0}^{n-1} \log \left|\phi^{i}\left(T^{j} x\right)\right| \leqslant m\right\}\right) \\
& -\mu\left(\left\{x:\left|\phi^{i}(x)-\phi(x)\right| \geqslant \delta\right\}\right) \\
\geqslant & \frac{1}{m}-\delta
\end{aligned}
$$

for $i$ large enough, we have

$$
\mu\left(\left\{x: \frac{1}{n} \sum_{j=0}^{n-1} \log \left|\phi\left(T^{j} k\right)\right| \leqslant m\right\}\right) \geqslant \frac{1}{m} .
$$

Thus $\phi$ is in $E_{m, N}^{c}$.

To find a space where the functions in which we are interested (those for which $(1 / n) \sum_{j=0}^{n-1} \log \left|\phi \circ T^{j}\right| \rightarrow-\infty$, that is $\left.\int_{X} \log |\phi| d \mu=-\infty\right)$ form a Baire space, we look at $L^{1}(X)$. For functions, $\phi$, in $L^{1}(X)$, since $\log ^{+}|\phi| \leqslant|\phi|, \int_{X} \log |\phi| d \mu=-\infty$ is equivalent to $\int_{X} \log ^{-}|\phi| d \mu=\infty$. The following theorem states that, for $\phi$ among $L^{1}(X)$ functions, $f_{x, \phi, T}$ typically has infinite order of growth.

THEOREM 1. Let $\left(b_{n}\right)$ be a sequence of positive real numbers such that $b_{n} / n \rightarrow \infty$. The set of functions $\phi$ in $L^{1}(X)$ that have $\phi(x) \neq 0$ a.e., $\int_{X} \log ^{-}|\phi| d \mu=\infty$, and

$$
\limsup _{n \rightarrow \infty} \frac{1}{b_{n}} \sum_{j=0}^{n-1} \log \left|\phi\left(T^{j} x\right)\right|=0 \quad \text { a.e. }
$$

is residual in $L^{1}(X)$.

Proof. Let $R=\left\{\phi \in L^{1}(x): \int_{X} \log ^{-}|\phi| d \mu=\infty\right\}$ and $S=\{\phi \in$ $\left.L^{1}(X): \mu(\{x: \phi(x)=0\})=0\right\}$. We can see that $R$ is residual in $L^{1}(X)$ by writing

$$
R=\bigcap_{k=1}^{\infty} R_{k}=\bigcap_{k=1}^{\infty}\left\{\phi \in L^{1}(X): \int_{X} \log ^{-}|\phi| d \mu>k\right\}
$$

and noting that each $R_{k}$ is open and dense in $L^{1}(X)$. Likewise, we can write

$$
S=\bigcap_{k=1}^{\infty} S_{k}=\bigcap_{k=1}^{\infty}\left\{\phi \in L^{1}(X): \mu(\{x: \phi(x)=0\})<\frac{1}{k}\right\}
$$

and see that each $S_{k}$ is open and dense in $L^{1}(X)$.

Now consider

$$
\begin{aligned}
\{\phi & \left.\in R \cap S: \limsup _{n \rightarrow \infty} \frac{1}{b_{n}} \sum_{j=0}^{n-1} \log \left|\phi\left(T^{j} x\right)\right|=0 \text { a.e. }\right\} \\
& =\left\{\phi \in R \cap S: \limsup _{n \rightarrow \infty} \frac{1}{b_{n}} \sum_{j=0}^{n-1} \log \left|\phi\left(T^{j} x\right)\right| \geqslant 0 \text { a.e. }\right\} .
\end{aligned}
$$


Let $d_{\text {meas }}$ denote any fixed metric for the topology of convergence in measure on $L^{1}(X)$. We have

$$
\begin{aligned}
& \left\{\phi \text { in } R \cap S: \limsup _{n \rightarrow \infty} \frac{1}{b_{n}} \sum_{j=0}^{n-1} \log \left|\phi\left(T^{j} x\right)\right| \geqslant 0 \text { a.e. }\right\} \\
& \supset\{\phi \text { in } R \cap S: \text { for every } \varepsilon>0 \text {, for every } N \text {, there exists } \\
& \left.n \geqslant N \text { such that } d_{\text {meas }}\left(\frac{1}{b_{n}} \sum_{j=0}^{n-1} \log \left|\phi \circ T^{j}\right|, 0\right)<\varepsilon\right\} \\
& =\bigcap_{m=1}^{\infty} \bigcap_{N=1}^{\infty} \bigcup_{n=N}^{\infty}\left\{\phi \text { in } R \cap S: d_{\text {meas }}\left(\frac{1}{b_{n}} \sum_{j=0}^{n-1} \log \left|\phi \circ T^{j}\right|, 0\right)<\frac{1}{m}\right\} \\
& =\bigcap_{m=1}^{\infty} \bigcap_{N=1}^{\infty} E_{m, N} .
\end{aligned}
$$

We will show that each $E_{m, N}$ is a dense open subset of $R \cap S$.

First, we fix any $\phi$ in $R \cap S$ and any $\varepsilon>0$. For each $k=1,2,3 \ldots$ define

$$
\phi_{k}(x)= \begin{cases}\phi(x) & \text { if }|\log | \phi(x)|| \leqslant k, \\ 1 & \text { otherwise. }\end{cases}
$$

Now

$$
\begin{aligned}
\lim _{k \rightarrow \infty} \mu\left(\left\{x: \phi(x) \neq \phi_{k}(x)\right\}\right)= & \lim _{k \rightarrow \infty} \mu\left(\left\{x:|\phi(x)|>e^{k}\right\}\right) \\
& +\lim _{k \rightarrow \infty} \mu\left(\left\{x:|\phi(x)|<e^{-k}\right\}\right) \\
= & 0+\mu\left(\bigcap_{k=1}^{\infty}\left\{x:|\phi(x)|<e^{-k}\right\}\right)=0 .
\end{aligned}
$$

Using this and the fact that $\phi$ is in $L^{1}(X)$, we can fix $k$ large enough that $\left\|\phi_{k}-\phi\right\|_{1}<\varepsilon$. Now $\log \left|\phi_{k}\right|$ is in $L^{1}(X)$, so

$$
\lim _{n \rightarrow \infty}\left|\frac{1}{n} \sum_{j=0}^{n-1} \log \right| \phi_{k}\left(T^{j} x\right)||=\left|\int_{X} \log \right| \phi_{k}|d \mu| \leqslant k \quad \text { a.e. }
$$

and thus

$$
\lim _{n \rightarrow \infty} \frac{1}{b_{n}} \sum_{j=0}^{n-1} \log \left|\phi_{k}\left(T^{j} x\right)\right|=\lim _{n \rightarrow \infty} \frac{n}{b_{n}} \frac{1}{n} \sum_{j=0}^{n-1} \log \left|\phi_{k}\left(T^{j} x\right)\right|=0 \quad \text { a.e. }
$$

Then we must also have

$$
\lim _{n \rightarrow \infty} \sum_{j=0}^{n-1} \log \left|\phi_{k}\left(T^{j} x\right)\right|=0 \quad \text { in measure, }
$$

so, for all large $n$,

$$
d_{\text {meas }}\left(\frac{1}{b_{n}} \sum_{j=0}^{n-1} \log \left|\phi_{k} \circ T^{j}\right|, 0\right)<\frac{1}{m}
$$


Therefore $\phi_{k}$ is in $E_{m, N}$. It is clear that

$$
E_{m, N}^{c}=\bigcap_{n=N}^{\infty}\left\{\phi \in R \cap S: d_{\text {meas }}\left(\frac{1}{b_{n}} \sum_{j=0}^{n-1} \log \left|\phi \circ T^{j}\right|, 0\right) \geqslant \frac{1}{m}\right\}
$$

must be closed in $R \cap S$ since $\left\{\psi \in R \cap S: d_{\text {meas }}(\psi, 0) \geqslant 1 / m\right\}$ is closed in the $d_{\text {meas }}$ topology in $R \cap S$.

3. Pseudo-lacunarity. A gap series (or lacunary series) is a power series in which there occur arbitrarily long gaps between successive nonzero coefficients. Although the power series for $f_{x, \phi, T}$ can never be a gap series since all its coefficients are nonzero, in order to have $\int_{X} \log |\phi| d \mu=-\infty$, the sequence $\phi(x), \phi(T x), \phi\left(T^{2} x\right), \ldots$ must contain values that are small enough often enough that the Cesáro means of $\log |\phi(x)|, \log |\phi(T x)|, \log \left|\phi\left(T^{2} x\right)\right|, \ldots$ diverge to $-\infty$ for almost every $x$. It seems reasonable, then, that the sequence of coefficients $\phi(x), \phi(x) \phi(T x)$, $\phi(x) \phi(T x) \phi\left(T^{2} x\right), \ldots$ contain arbitrarily long stretches of terms that are much smaller than the surrounding terms. In this sense $f_{x, \phi, T}$ resembles a gap series. Some of the value distribution properties of lacunary series also hold for these "pseudolacunary" series $f_{x, \phi, T}$. The following theorem is an exact analogue of a theorem of Pólya for gap series (see Saks and Zygmund [8, p. 337]).

THEOREM 2. If $\phi(x) \neq 0$ a.e., $\int_{X} \log |\phi| d \mu=-\infty$, and $f_{x, \phi, T}$ is of finite order for almost every $x$, then for almost every $x, f_{x, \phi, T}$ assumes every finite value an infinite number of times.

We will need the following lemma.

Lemma 1. Let $\phi(x) \neq 0$ a.e. and $\int_{X} \log |\phi| d \mu=-\infty$. Then almost every $x$ in $X$ has the following property: If $c_{0}, c_{1}, \ldots, c_{r}$ are any complex numbers, not all zero, then there are arbitrarily large values of $k$ for which $c_{0}+c_{1} \phi\left(T^{k+1} x\right)+c_{2} \phi\left(T^{k+1} x\right) \phi\left(T^{k+2} x\right)+\cdots+c_{r} \phi\left(T^{k+1} x\right) \cdots \phi\left(T^{k+r} x\right) \neq 0$.

The lemma asserts that, for infinitely many values of $k$, the point

$$
q_{k}=\left(\phi\left(T^{k+1} x\right), \phi\left(T^{k+1} x\right) \phi\left(T^{k+2} x\right), \ldots, \phi\left(T^{k+1} x\right) \cdots \phi\left(T^{k+r} x\right)\right)
$$

lies off of the hyperplane of zeros of $c_{0}+c_{1} \alpha_{1}+c_{2} \alpha_{2}+\cdots+c_{r} \alpha_{r}$.

Proof (OF THE LeMMA). Fix any $x$ for which

$$
\lim _{n \rightarrow \infty} \frac{1}{n} \sum_{j=0}^{n-1} \log \left|\phi\left(T^{j} x\right)\right|=-\infty
$$

and suppose that

$$
c_{0}+c_{1} \phi\left(T^{k+1} x\right)+\cdots+c_{r} \phi\left(T^{k+1} x\right) \cdots \phi\left(T^{k+r} x\right)=0
$$

for every $k \geqslant N$. Without loss of generality, we may assume $c_{0} \neq 0$ and $c_{r} \neq 0$. Now there must exist a positive constant, $M$, depending only on $c_{0}, c_{1}, \ldots, c_{r}$, such that for every $k \geqslant N$ at least one of $\log \left|\phi\left(T^{k+1} x\right)\right|, \log \left|\phi\left(T^{k+1} x\right)\right|+$ $\log \left|\phi\left(T^{k+2} x\right)\right|, \ldots, \log \left|\phi\left(T^{k+1} x\right)\right|+\cdots+\log \left|\phi\left(T^{k+r} x\right)\right|$ is larger than $\log M$. This 
implies that

$$
\begin{aligned}
\frac{1}{n} \sum_{j=0}^{n-1} \log \left|\phi\left(T^{j} x\right)\right|> & \frac{1}{n} \sum_{j=0}^{N} \log \left|\phi\left(T^{j} x\right)\right|+\frac{1}{n} k \log M \\
& +\frac{1}{n} \sum_{j=n-i}^{n-1} \log \left|\phi\left(T^{j} x\right)\right|,
\end{aligned}
$$

where $k$ satisfies $n-N-1 \geqslant k \geqslant(n-N-1-i) / r \geqslant(n-N-1-r) / r$ and $i$ is less than $r$. Thus

$$
\begin{aligned}
& \lim _{n \rightarrow \infty} \frac{1}{n} \sum_{j=0}^{n-1} \log \left|\phi\left(T^{j} x\right)\right| \\
& \geqslant \underset{n \rightarrow \infty}{\limsup }\left[\frac{1}{n} \sum_{j=0}^{N} \log \left|\phi\left(T^{j} x\right)\right|\right. \\
& \left.+\frac{1}{n} \frac{n-N-1-r}{r} \log M+\frac{1}{n} \sum_{j=n-i}^{n-1} \log \left|\phi\left(T^{j} x\right)\right|\right] \\
& =0+\frac{1}{r} \log M+0 .
\end{aligned}
$$

We now have contradicted the choice of $x$ as a point where the ergodic averages diverge to $-\infty$.

Proof (of THE THEOREM). Let us fix any $x$ in $X$ for which $f_{x, \phi, T}$ is nonconstant and has finite order and suppose that $f_{x, \phi, T}$ takes on the value $w$ only finitely many times. We can write

$$
f_{x, \phi, T}(z)-w=P(z) e^{h(z)},
$$

where $P$, the canonical product of $f_{x, \phi, T}-w$, is a polynomial. By Hadamard's Theorem, $h$ is also a polynomial. Now we have

$$
\begin{aligned}
& f_{x, \phi, T}^{\prime}(z)=P^{\prime}(z) e^{h(z)}+P(z) h^{\prime}(z) e^{h(z)}, \\
& z P(z) f_{x, \phi, T}^{\prime}(z)=z P^{\prime}(z) P(z) e^{h(z)}+z P(z) h^{\prime}(z) P(z) e^{h(z),} \\
& \left(z f_{x, \phi, T}^{\prime}(z)\right) P(z)=\left(f_{x, \phi, T}(z)-w\right)\left[z P^{\prime}(z)+z P(z) h^{\prime}(z)\right],
\end{aligned}
$$

or, defining $Q(z)={ }_{z} P^{\prime}(z)+z P(z) h^{\prime}(z)$,

$$
\left(z f_{x, \phi, T}^{\prime}(z)\right) P(z)=\left(f_{x, \phi, T}(z)-w\right) Q(z) .
$$

Writing

$$
f_{x, \phi, T}(z)=\sum_{k=0}^{\infty} a_{k} z^{k}, \quad P(z)=\sum_{i=0}^{r} p_{i} z^{i}, \quad \text { and } \quad Q(z)=\sum_{i=0}^{r} q_{i} z^{i},
$$

we have

$$
\left(\sum_{k=0}^{\infty} k a_{k} z^{k}\right)\left(\sum_{i=0}^{r} p_{i} z^{i}\right)=\left(\sum_{k=0}^{\infty} a_{k} z^{k}\right)\left(\sum_{i=0}^{r} q_{i} z^{i}\right)-w \sum_{i=0}^{r} q_{i} z^{i} .
$$


Looking at the coefficient of $z^{n}$ for $n>r$ we have

$$
\begin{gathered}
\sum_{i=0}^{r}(n-i) a_{n-i} p_{i}=\sum_{i=0}^{r} a_{n-i} q_{i} \text { for } n>r, \\
n \sum_{i=0}^{r} a_{n-i} p_{i}=\sum_{i=0}^{r} a_{n-i}\left(q_{i}+i p_{i}\right) \text { for } n>r, \text { and } \\
\frac{\sum_{i=0}^{r} a_{n-i}\left(q_{i}+i p_{i}\right)}{\sum_{i=0}^{r} a_{n-i} p_{i}}=n \text { for } n>r \text { when } \sum_{i=0}^{r} a_{n-i} p_{i} \neq 0 .
\end{gathered}
$$

Recalling that $a_{k}=\phi(x) \phi(T x) \cdots \phi\left(T^{k-1} x\right)$ and that $\phi$ is nonvanishing, we have

$$
\frac{\sum_{i=0}^{r}\left(q_{i}+i p_{i}\right) \phi\left(T^{n-r} x\right) \cdots \phi\left(T^{n-i-1} x\right)}{\sum_{i=0}^{r} p_{i} \phi\left(T^{n-r} x\right) \cdots \phi\left(T^{n-i-1} x\right)}=n \text { for } n>r
$$

when $\sum_{i=0}^{r} p_{i} \phi\left(T^{n-r} x\right) \cdots \phi\left(T^{n-i-1} x\right) \neq 0$; or equivalently,

$$
\frac{\sum_{i=0}^{r}\left(q_{i}+i p_{i}\right) \phi\left(T^{k+1} x\right) \cdots \phi\left(T^{k+r-i} x\right)}{\sum_{i=0}^{r} p_{i} \phi\left(T^{k+1} x\right) \cdots \phi\left(T^{k+r-i} x\right)}=r+k+1 \text { for } k \geqslant 0
$$

when $\sum_{i=0}^{r} p_{i} \phi\left(T^{k+1} x\right) \cdots \phi\left(T^{k+r-i} x\right) \neq 0$.

We now define a rational function $H: \mathbf{C}^{r} \rightarrow \mathbf{C}$ by

$$
H\left(\alpha_{1}, \ldots, \alpha_{r}\right)=\frac{\sum_{i=0}^{r}\left(q_{i}+i p_{i}\right) \alpha_{1} \cdots \alpha_{r-i}}{\sum_{i=0}^{r} p_{i} \alpha_{1} \cdots \alpha_{r-i}},
$$

so that we have $H\left(\phi\left(T^{k+1} x\right), \ldots, \phi\left(T^{k+r} x\right)\right)=r+k+1$ whenever $k \geqslant 0$ and $H$ is defined at $\left(\phi\left(T^{k+1} x\right), \ldots, \phi\left(T^{k+r} x\right)\right)$.

The Poincaré Recurrence Theorem implies that almost every $x$ in $X$ has the following property: For any $r=1,2,3, \ldots$ and any point, $z$, in $\mathbf{C}^{r}$, there exists an arbitrarily small neighborhood of $z$ such that if there is any value of $k \geqslant 0$ with $\left(\phi\left(T^{k+1} x\right), \ldots, \phi\left(T^{k+r} x\right)\right)$ in this neighborhood, then there are infinitely many such values of $k$. Now suppose we have chosen an $x$ with this property and which satisfies the result of Lemma 1 . Then we have a $k_{0} \geqslant 0$ with

$$
\sum_{i=0}^{r} p_{i} \phi\left(T^{k_{0}+1} x\right) \cdots \phi\left(T^{k_{0}+r-i} x\right) \neq 0
$$

so $H\left(\phi\left(T^{k_{0}+1} x\right), \ldots, \phi\left(T^{k_{0}+r} x\right)\right)=r+k_{0}+1$. Now we can choose a neighborhood, $U$, of $\left(\phi\left(T^{k_{0}+1} x\right), \ldots, \phi\left(T^{k_{0}+r} x\right)\right)$ in $C^{r}$ small enough that $H$ is defined (and continuous) on $U$ and that

$$
\left|H(y)-\left(r+k_{0}+1\right)\right|<\frac{1}{2}
$$

for every $y$ in $U$. But we know that there are infinitely many values of $k \geqslant 0$ with $\left(\phi\left(T^{k+1} x\right), \ldots, \phi\left(T^{k+r} x\right)\right)$ in $U$. This means that, for values of $k>k_{0}$, we have both that

$$
\left|H\left(\phi\left(T^{k+1} x\right), \ldots, \phi\left(T^{k+r} x\right)\right)-\left(r+k_{0}+1\right)\right|<\frac{1}{2}
$$

and that $H\left(\phi\left(T^{k+1} x\right), \ldots, \phi\left(T^{k+r} x\right)\right)=r+k+1$, which is contradictory. 
4. Conditions for rationality. We find that, for finite radius of convergence, it is more natural to first study the series

$$
g_{x, \phi, T}(z)=\sum_{n=0}^{\infty} \phi\left(T^{n} x\right) z^{n} .
$$

While working with this series, we will assume that $\phi$ is in $L^{1}(X)$, for then $T$ ergodic implies that $g_{x, \phi, T}$ has radius of convergence one almost everywhere (see Ionescu Tulcea [4]). We will prove the following lemma for $g_{x, \phi, T}$ only, but the proof of the corresponding statement for $f_{x, \phi, T}$ is analogous.

LEMMA 2. $g_{x, \phi, T}$ represents a rational function for almost every $x$ if and only if an equation of the form

$$
a_{p} \phi(x)+a_{p-1} \phi(T x)+\cdots+a_{0} \phi\left(T^{p} x\right)=0
$$

is satisfied almost everywhere.

Proof. For fixed $T$ and $\phi, f_{x, \phi . T}$ will be rational if and only if there are polynomials

$$
P_{x}(z)=\sum_{i=0}^{p(x)} a_{i}(x) z^{i} \text { and } Q_{x}(z)=\sum_{i=0}^{q(x)} b_{i}(x) z^{i}
$$

such that $P_{x}(z) g_{x, \phi, T}(z)=Q_{x}(z)$. We will assume that $P_{x}(z)$ is of minimal degree and monic. By the identity theorem,

$$
\sum_{i+j=n} a_{i}(x) \phi\left(T^{j} x\right)=0 \text { for } n>q(x) .
$$

so

$$
a_{p(x)}(x) \phi\left(T^{n-p(x)} x\right)+\cdots+a_{1}(x) \phi\left(T^{::-1} x\right)+a_{0}(x) \phi\left(T^{n} x\right)=0
$$

for $n \geqslant \max \{p(x), q(x)+1\}$ for almost every $x$.

Now

$$
\begin{aligned}
g_{x, \phi, T}(z) & =\sum_{n=0}^{\infty} \phi\left(T^{n} x\right) z^{n}=\phi(x)+\sum_{n=1}^{\infty} \phi\left(T^{n} x\right) z^{n} \\
& =\phi(x)+\sum_{n=0}^{\infty} \phi\left(T^{n+1} x\right) z^{n+1}=\phi(x)+z g_{T x, \phi, T}(z),
\end{aligned}
$$

from which it is clear that $g_{x, \phi, T}$ and $g_{T x, \phi, T}$ have precisely the same poles.

For any integer $n \geqslant 0$, consider the grid of lines in $\mathbf{C}$

$$
\left\{\operatorname{Re}(z)=k / 2^{n}\right\}_{k=-\infty}^{\infty} \cup\left\{\operatorname{Im}(z)=k / 2^{n}\right\}_{k=-\infty}^{\infty} .
$$

Let $\mathscr{C}_{n}$ be the countable set of all simple closed curves made up of segments of the lines in this grid. For each curve, $C$, in $\mathscr{C}=\cup_{n} \mathscr{C}_{n}$ we define the function $I_{C}(x)$ on $X$ by

$$
I_{C}(x)=\frac{1}{2 \pi i} \int_{C} \frac{g_{x, \phi, T}^{\prime}(z)}{g_{x, \phi, T}(z)} d z
$$


$I_{C}(x)=\infty$ precisely when one or more poles or zeros of $g_{x, \phi, T}$ lie on the curve $C$ and otherwise is the number of zeros minus the number of poles of $g_{x, \phi, T}$ that lie in the region enclosed by $C$. Now, defining

$$
I_{C}^{*}(x)= \begin{cases}I_{C}(x) & \text { if } I_{C}(x) \neq \infty, \\ 0 & \text { if } I_{C}(x)=\infty,\end{cases}
$$

we have $q(x)=\sup _{C \in \mathscr{C}} I_{C}^{*}(x)$ and $p(x)=-\inf _{C \in \mathscr{C}} I_{C}^{*}(x)$. We can see by these formulations that $p(x)$ and $q(x)$ must be measurable functions of $x$. We have already seen that $p(x)$ is constant along orbits, so we now have $p(x)=p$ a.e. By extending this technique and considering functions $-\inf _{C \in \mathscr{C}_{U}} I_{C}(x)$, where $\mathscr{C}_{U}$ is the set of those curves in $\mathscr{C}$ which lie within a fixed region $U$, we find that the poles of $g_{x, \phi, T}$ are not only constant along orbits, but must be the same for almost every $x$. Thus, each $a_{i}(x)$ is constant almost everywhere. Using $g_{x, \phi, T}(z)=\phi(z)+$ $z g_{T x, \phi, T}(z)$ and $P_{T x}=P_{x}$, we have

$$
\frac{Q_{x}(z)}{P_{x}(z)}=\phi(x)+z \frac{Q_{T x}(z)}{P_{x}(z)} \quad \text { and } \quad Q_{T x}(z)=\frac{Q_{x}(z)-\phi(x) P_{x}(z)}{z} .
$$

It is clear from this that $q\left(T^{n} x\right)$ is bounded by $p-1$ for sufficiently large $n$, and, thus, $q(x) \leqslant p-1$ a.e. This gives that $a_{p} \phi\left(T^{n-p} x\right)+\cdots+a_{1} \phi\left(T^{n-1} x\right)+$ $a_{0} \phi\left(T^{n} x\right)=0$ a.e. for $n \geqslant p$, which implies $a_{p} \phi(x)+\cdots+a_{1} \phi\left(T^{p-1} x\right)+$ $a_{0} \phi\left(T^{p} x\right)=0$ a.e.

The converse is straightforward.

We denote the density of a subset, $J$, of the natural numbers by $\delta(J)$ and the upper and lower densities by $\bar{\delta}(J)$ and $\underline{\delta}(J)$.

LemMa 3. Let $\left(a_{j}\right)$ be an almost periodic sequence in $\mathbf{C}$. Suppose there is a subset, $J$, of the natural numbers such that $\bar{\delta}(J)>0$ and $\lim _{j \rightarrow \infty, j \in J} a_{j}=0$. Then $J$ has $a$ subset, $K$, such that $\delta(K)=0$ and $a_{j}=0$ for every $j$ in $J \backslash K$.

Proof. Let us write $a_{j}=f\left(R^{j} g\right)$, where $R$ is an ergodic rotation of a compact abelian group and $f$ is a continuous function on that group. We denote the ball in $\mathbf{C}$ of radius $\varepsilon$ about 0 by $B(0 ; \varepsilon)$. Let $J^{\prime}=J \cup\left\{j \in \mathbf{N}: a_{j}=0\right\}$. Using $\lim _{j \rightarrow \infty, j \in J^{\prime}} a_{j}=0$ and the Ergodic Theorem, we have

$$
\begin{aligned}
\mu\left(f^{-1}(B(0 ; \varepsilon))\right) & =\limsup _{n \rightarrow \infty} \frac{1}{n} \sum_{j=0}^{n-1} \chi_{f^{-1}(B(0 ; \varepsilon))}\left(R^{j} g\right) \\
& =\limsup _{n \rightarrow \infty} \frac{1}{n} \sum_{j=0}^{n-1} \chi_{B(0 ; \varepsilon)}\left(a_{j}\right) \\
& \geqslant \limsup _{n \rightarrow \infty} \frac{1}{n} \sum_{j=0}^{n-1} \chi_{J^{\prime}}(j)=\bar{\delta}\left(J^{\prime}\right) .
\end{aligned}
$$

Now

$$
\begin{aligned}
\mu\left(f^{-1}\{0\}\right) & =\mu\left(\bigcap_{i=1}^{\infty} f^{-1}\left(B\left(0 ; \frac{1}{i}\right)\right)\right) \\
& =\lim _{i \rightarrow \infty} \mu\left(f^{-1}\left(B\left(0 ; \frac{1}{i}\right)\right)\right) \geqslant \bar{\delta}\left(J^{\prime}\right) .
\end{aligned}
$$


Then

$$
\mu\left(f^{-1}\{0\}\right) \geqslant \bar{\delta}\left(J^{\prime}\right) \geqslant \bar{\delta}\left\{j \in \mathbf{N}: a_{j}=0\right\}=\mu\left(f^{-1}\{0\}\right),
$$

so $\delta\left(J^{\prime}\right)$ exists and $\mu\left(f^{-1}\{0\}\right)=\delta\left(J^{\prime}\right)$.

Now let $K=\left\{j \in J: a_{j} \neq 0\right\}=\left\{j \in J^{\prime}: a_{j} \neq 0\right\}$. We have

$$
\frac{1}{n} \sum_{j=0}^{n-1} \chi_{J^{\prime}}(j)=\frac{1}{n} \sum_{j=0}^{n-1} \chi_{K}(j)+\frac{1}{n} \sum_{j=0}^{n-1} \chi_{\left\{j: a_{j}=0\right\}}(j) \text {, }
$$

which implies $\delta(K)$ exists and equals zero.

THEOREM 3. Suppose $\phi$ is in $L^{1}(X)$. Then $g_{x, \phi, T}$ represents a rational function if and only if $\phi$ is a finite sum of eigenfunctions of $T$. In this case, the poles are all simple and occur at the inverses of the eigenvalues corresponding to these eigenfunctions.

Proof. If $\phi(x)=0$ a.e., the result is trivial; henceforth, we will assume that $\phi(x)=0$ does not hold almost everywhere and $g_{x, \phi, T}$, therefore, has radius of convergence one. The condition of Lemma 2 is equivalent to

$$
a_{p} \phi\left(T^{n} x\right)+a_{p-1} \phi\left(T^{n+1} x\right)+\cdots+a_{0} \phi\left(T^{n+p} x\right)=0 \text { a.e. for every } n .
$$

This liner difference equation for the sequence $\left(\phi\left(T^{n} x\right)\right)$ has a unique solution of the form

$$
\begin{aligned}
\phi\left(T^{n} x\right)= & {\left[c_{11}(x)+n c_{12}(x)+n^{2} c_{13}(x)+\cdots+n^{n_{1}-1} c_{1 n_{1}}(x)\right] m_{1}^{n} } \\
& +\left[c_{21}(x)+n c_{22}(x)+n^{2} c_{23}(x)+n^{n_{2}-1} c_{2 n_{2}}(x)\right] m_{2}^{n} \\
& +\cdots+\left[c_{k 1}(x)+n c_{k 2}(x)+n^{2} c_{k 3}(x)+\cdots+n^{n_{k}-1} c_{k n_{k}}(x)\right] m_{k}^{n} \quad \text { a.e. }
\end{aligned}
$$

where $m_{1}, m_{2}, \ldots, m_{k}$ are the distinct roots of

$$
\hat{P}_{\phi}(z)=a_{p}+a_{p-1} z+\cdots+a_{0} z^{P},
$$

with each $m_{j}$ occurring as a root of multiplicity $n_{j}$. It is clear that this new equation is satisfied (with all $n_{i}=1$ ) when $\phi$ is a finite sum of eigenfunctions. For the converse, we will show that

$$
\phi\left(T^{x} n\right)=\sum_{j=1}^{k}\left[c_{j 1}(x)+n c_{j 2}(x)+n^{2} c_{j 3}(x)+\cdots+n^{n_{j}-1} c_{j n_{j}}(x)\right] m_{j}^{n} \quad \text { a.e. }
$$

implies that, for each $j, n_{j}=1$ and $c_{j 1}(x)$ is an eigenfunction of $T$ with corresponding eigenvalue $m_{j}$.

First, we note that the poles of $g_{x, \phi, T}$ are

$$
z=\frac{1}{m_{1}}, z=\frac{1}{m_{2}}, \ldots, z=\frac{1}{m_{k}} .
$$

This is true because $m_{1}, \ldots, m_{k}$ are roots of $\hat{P}_{\phi}(z)=z^{p} P_{\phi}(1 / z)$, where the roots of $P_{\phi}(z)=a_{p} z^{p}+a_{p-1} z^{p-1}+\cdots+a_{0}$ are precisely the poles of $g_{x, \phi, T}$, as in the proof of Lemma 2. Since the radius of convergence of $g_{x, \phi, T}$ is one, we have $\left|m_{j}\right| \leqslant 1$ for each $j$. We will suppose that $\left|m_{j}\right|<1$ for some $j$ and see that this leads to a contradiction. 
We write

$$
m_{j}=r_{j} e^{i \theta_{j}}, \quad r_{j}>0 .
$$

for each $j$, and assume the roots are ordered so that $r_{1}=r_{2}=\cdots=r_{h}<r_{h+1} \leqslant$ $\cdots \leqslant r_{k} \leqslant 1$ and $n_{1}=n_{2}=\cdots=n_{g}>n_{g+1} \geqslant \cdots \geqslant n_{h}$ (possibly $g=h$ ). Now (1) $\phi\left(T^{n} x\right)=r_{1}^{n}\left[n^{n_{1}-1} \sum_{j=1}^{g} c_{j n_{1}}(x) e^{i n \theta_{j}}\right.$

$$
\begin{gathered}
+\sum_{j=1}^{g}\left[c_{j 1}(x)+n c_{j 2}(x)+\cdots+n^{n_{1}-2} c_{j n_{1}-1}(x)\right] e^{i n \theta_{j}} \\
\left.+\sum_{j=g+1}^{h}\left[c_{j 1}(x)+n c_{j 2}(x)+\cdots n^{n_{j}-1} c_{j n_{j}}(x)\right] e^{i n \theta_{j}}\right] \\
+\sum_{j=h+1}^{k}\left[c_{j 1}(x)+n c_{j 2}(x)+\cdots+n^{n_{j}-1} c_{j n_{j}}(x)\right] r_{j}^{n} e^{i n \theta_{j}} \quad \text { a.e. }
\end{gathered}
$$

Let $D$ be any bounded region in $\mathbf{C}$ with $\mu\left(\phi^{-1}(D)\right)>0$. We now fix any $x$ for which (1) holds and which has

$$
\lim _{n \rightarrow \infty} \frac{1}{n} \sum_{j=0}^{n-1} \chi_{\phi^{-1}(D)}\left(T^{-j} x\right)=\mu\left(\phi^{-1}(D)\right) .
$$

Since $r_{1}<r_{j}$ for all $j=h+1, \ldots, k$, the first of the two terms of (1) must dominate when $n \leqslant N<0$. This means that $\phi\left(T^{n} x\right)$ for $n \leqslant N$ can be in $D$ only when

$$
\begin{aligned}
r_{1}^{n} \mid n^{n_{1}-1} \sum_{j=1}^{g} c_{j n_{1}}(x) e^{i n \theta_{j}} & \\
& +\sum_{j=1}^{g}\left[c_{j 1}(x)+n c_{j 2}(x)+\cdots+n^{n_{1}-2} c_{j n_{1}-1}(x)\right] e^{i n \theta_{j}} \\
& +\sum_{j=g+1}^{h}\left[c_{j 1}(x)+n c_{j 2}(x)+\cdots+n^{n_{j}-1} c_{j n_{j}}(x)\right] e^{i n \theta_{j}} \mid \\
\leqslant & C_{1} n^{s} r_{h+1}^{n},
\end{aligned}
$$

where $C_{1}$ is a constant depending on $D$ and on $c_{j 1}(x), \ldots, c_{j n_{j}}(x), j=h+1, \ldots, k$. This inequality implies

$$
\left|\sum_{j=1}^{g} c_{j n_{1}}(x) e^{i n \theta_{j}}\right| \leqslant C_{1} n^{v}\left(\frac{r_{h+1}}{r_{1}}\right)^{n}+\frac{C_{2} n^{n_{1}-2}}{n^{n_{1}-1}},
$$

where $C_{2}$ is a constant depending on $c_{j 1}(x), \ldots, c_{j n_{1}-1}(x), j=1, \ldots, g$, and on $c_{j 1}(x), \ldots, c_{j n_{1}}(x), j=g+1, \ldots, h$. Now the right-hand side of (2) tends to zero as $n$ tends to $-\infty$, and we have that $\phi\left(T^{n} x\right)$ is in $D$ only if (2) holds.

Since $\mu\left(\phi^{-1}(D)\right)>0$, the set of $n$ for which $\phi\left(T^{-n} x\right)$ is in $D$, and therefore the set of $n$ such that (2) holds for $-n$, must have positive density in the natural numbers. Noting that the left-hand side of (2) is an almost periodic sequence, we apply 
Lemma 3. This tells us that there is a set, $I$, of indices such that the left-hand side of (2) is zero for every $n$ in $I$ and that

$$
\begin{aligned}
\underline{\delta}(I) & \geqslant \underline{\delta}(\{n:(2) \text { holds for }-n\}) \\
& \geqslant \delta\left(\left\{n: \phi\left(T^{-n} x\right) \text { is in } D\right\}\right)=\mu\left(\phi^{-1}(D)\right) .
\end{aligned}
$$

Since $\mu\left(\phi^{-1}\{\infty\}\right)=0$, we can choose the region $D$ with $0<\mu\left(\phi^{-1}(D)\right) \leqslant \underline{\delta}(I)$, unless $\underline{\delta}(I)=1$. Thus, the only way to avoid a contradiction is for $\underline{\delta}(I)=1$, which means that the left-hand side of (2) is zero except possibly for $n$ 's making up a set of density zero. By almost periodicity, then, this sum must be zero for every $n$. Therefore, we can write

$$
\begin{aligned}
\phi\left(T^{n} x\right)= & \sum_{j=1}^{g}\left[c_{j 1}(x)+n c_{j 2}(x)+\cdots+n^{n_{j}-2} c_{j n_{j}-1}(x)\right] m_{j}^{n} \\
& +\sum_{j=g+1}^{k}\left[c_{j 1}(x)+n c_{j 2}(x)+\cdots+n^{n_{j}-1} c_{j n_{j}}(x)\right] m_{j}^{n},
\end{aligned}
$$

which contradicts the uniqueness of the solution to the difference equation. It must, therefore, be true that all $r_{j}=1$.

We can show that all the roots must have multiplicity one in a similar manner. Thus,

$$
\phi\left(T^{n} x\right)=c_{1}(x) e^{i n \theta_{1}}+c_{2}(x) e^{i n \theta_{2}}+\cdots+c_{k}(x) e^{i n \theta_{k}} \quad \text { a.e. }
$$

for every $n$, with $e^{i \theta_{1}}, \ldots, e^{i \theta_{k}}$ distinct. We need to show that each $c_{j}$ is an eigenfunction of $T$ with eigenvalue $e^{i \theta_{j}}$. For almost every $x, \phi\left(T^{n} x\right)=\phi\left(T^{n-1} T x\right)$, so

$$
\begin{aligned}
& c_{1}(x) e^{i n \theta_{1}}+\cdots+c_{k}(x) e^{i n \theta_{k}} \\
& \quad=c_{1}(T x) e^{i(n-1) \theta_{1}}+\cdots+c_{k}(T x) e^{i(n-1) \theta_{k}}
\end{aligned}
$$

for every $n$. This implies that

$$
\left[c_{1}(x) e^{i \theta_{1}}-c_{1}(T x)\right] e^{i(n-1) \theta_{1}}+\cdots+\left[c_{k}(x) e^{i \theta_{k}}-c_{k}(T x)\right] e^{i(n-1) \theta_{k}}=0
$$

for every $n$; that is, every vector in

$$
A=\left\{\left(e^{i(n-1) \theta_{1}}, \ldots, e^{i(n-1) \theta_{k}}\right): n \text { in } \mathbf{Z}\right\}
$$

is perpendicular to the vector

$$
V=\left(c_{1}(x) e^{i \theta_{1}}-c_{1}(T x), \ldots, c_{k}(x) e^{i \theta_{k}}-c_{k}(T x)\right)
$$

in $\mathbf{C}^{k}$. Therefore, supposing $V \neq 0$, the $k$ vectors $\left\{\left(e^{i n \theta_{1}}, \ldots, e^{i n \theta_{k}}\right): n=0,1, \ldots, k\right.$ -1 ) must be linearly dependent. Thus, there exist coefficients, $b_{n}, n=0,1, \ldots, k$ -1 , such that

$$
b_{0}+b_{1} e^{i \theta_{j}}+\cdots+b_{k-1} e^{i(k-1) \theta_{j}}=0
$$

for each $j=1,2, \ldots, k$. This means that each of the $k$ values $e^{i \theta_{j}}, j=1,2, \ldots, k$, is a root of the $k-1$ degree polynomial $b_{0}+b_{1} z+\cdots+b_{k-1} z^{k-1}=0$, which is a contradiction. Thus, we must have $V=0$ and

$$
c_{j}(T x)=c_{j}(x) e^{i \theta_{j}}
$$

for $j=1,2, \ldots, k$. 
In studying those series $f_{x, \phi, T}$ which represent rational functions, we assume that $\int_{X} \log |\phi| d \mu=0$, so that the radius of convergence is one.

The series $f_{x, \phi, T}$ can, under certain conditions, be viewed as a special type of series of the form of $g_{x, \phi, T}$, as follows. Let $S: X \times \mathbf{C} \rightarrow X \times \mathbf{C}$ be the skew product transformation defined by $S(x, w)=(T x, \phi(x) w)$. Then we have

$$
S^{n}(x, w)=\left(T^{n} x, \phi(x) \phi(T x) \cdots \phi\left(T^{n-1} x\right) w\right) .
$$

Let $\psi: X \times \mathbf{C} \rightarrow \mathbf{C}$ be given by $\psi(x, w)=w$. Now, for any $w$ in $\mathbf{C}$,

$$
\begin{aligned}
g_{(x, w), \psi, S}(z) & =\sum_{n=0}^{\infty} \psi\left(S^{n}(x, w)\right) z^{n} \\
& =w \sum_{n=0}^{\infty} \phi(x) \cdots \phi\left(T^{n-1} x\right) z^{n}=w f_{x, \phi, T}(z) .
\end{aligned}
$$

To assure that $S$ is measure-preserving, we need to assume $|\phi(x)|=1$ a.e. Then $S$ (which now acts on the unit circle, $S^{1}$ ) is ergodic if and only if there do not exist an integer $p \neq 0$ and a measurable function $h: X \rightarrow S^{1}$, such that

$$
[\phi(x)]^{p}=h(x) / h(T x) \text { a.e. }
$$

(see Anzai [3]).

COROllary 1. Suppose that $|\phi(x)|=1$ a.e. and that there do not exist an integer $p \neq 0$ and a measurable function $h: X \rightarrow S^{1}$, such that

$$
[\phi(x)]^{p}=h(x) / h(T x) \text { a.e. }
$$

Then $f_{x, \phi, T}$ represents a rational function for almost every $x$ if and only if $\phi$ is constant.

Proof. Suppose first that $f_{x, \phi, T}$ is rational. Since

$$
f_{x, \phi, T}(z)=\frac{1}{w} g_{\left(x, x^{\prime}\right), \psi, S}(z)
$$

we can, by Theorem 3, write

$$
\psi(x, w)=w=\sum_{i=1}^{k} c_{i}(x, w) \text { a.e. }(x, w),
$$

where each $c_{i}$ is an eigenfunction of $S$. For each $i, \lambda_{i}$ can be the eigenvalue associated with $c_{i}$ if and only if there exist an integer $p_{i}$ and a measurable function $h_{i}: X \rightarrow S^{1}$, such that

$$
[\phi(x)]^{p_{i}}=\lambda_{i} h_{i}(x) / h_{i}(T x) \text { a.e. }
$$

(see Anzai [3]).

Now $g_{i}(x, w)=w^{p_{i}} h_{i}(x)$ is an eigenfunction of $S$ with eigenvalue $\lambda_{i}$, so we can write $c_{i}(x, w)=a_{i} w^{p} h_{i}(x)$ a.e. $(x, w)$. We now have, for almost every $x$ and $w$ and for each $n$,

$$
\phi(x) \cdots \phi\left(T^{n-1} x\right) w=\psi\left(S^{n}(x, w)\right)=\sum_{i=1}^{k} a_{i} \lambda_{i}^{n} w^{p_{i}} h_{i}(x),
$$

so

$$
\phi(x) \cdots \phi\left(T^{n-1} x\right)=\sum_{i=1}^{k} a_{i} \lambda_{i}^{n} w^{p_{i}-1} h_{i}(x)
$$


Viewing this as a series in $w$, we must have

$$
\sum_{i \text { with } p_{i}=q} a_{i} \lambda_{i}^{n} h_{i}(x)=0
$$

for each $q \neq 1$. Thus, we may assume that all $p_{i}=1$, so we have

$$
\phi(x) \cdots \phi\left(T^{n-1} x\right)=\sum_{i=1}^{k} a_{i} \lambda_{i}^{n} h_{i}(x) \quad \text { a.e. }(x, w)
$$

for every $n$.

We see next that $k$ must equal one. First, we note that none of $\lambda_{1}, \lambda_{2}, \ldots, \lambda_{k}$ can be roots of unity, for if $\lambda_{i}^{q_{i}}=1, q_{i} \neq 0$, then

$$
\phi(x)=\lambda_{i} \frac{h_{i}(x)}{h_{i}(T x)} \quad \text { a.e. }
$$

implies

$$
[\phi(x)]^{q_{i}}=\lambda_{i}^{q_{i}} \frac{h_{i}^{q_{i}}(x)}{h_{i}^{q_{i}}(T x)}=\frac{h_{i}^{q_{i}}(x)}{h_{i}^{q_{i}}(T x)} \quad \text { a.e., }
$$

which contradicts one of the assumptions of the corollary. Now we can find rationally independent $\beta_{1}, \beta_{2}, \ldots, \beta_{m}$ in $S^{1}$ and integers, $P_{i, 1}, P_{i, 2}, \ldots, P_{i, m}$ for each $i=1,2, \ldots, k$ such that

$$
\lambda_{i}=\beta_{1}^{P_{i, 1}} \beta_{2}^{P_{i, 2}} \cdots \beta_{m}^{P_{i, m}}
$$

for every $i$. We now have

$$
\phi(x) \cdots \phi\left(T^{n-1} x\right)=\sum_{i=1}^{k} a_{i}\left(\beta_{1}^{P_{i, 1}} \cdots \beta_{m}^{P_{i, m}}\right)^{n} h_{i}(x) \text { a.e. }
$$

for every $n$. Next, fix any $x$ for which this equation holds. We define a measurepreserving transformation $R: S^{1} \times \cdots \times S^{1} \rightarrow S^{1} \times \cdots \times S^{1}$ by $R\left(\alpha_{1}, \ldots, \alpha_{m}\right)=$ $\left(\alpha_{1} \beta_{1}, \ldots, \alpha_{m} \beta_{m}\right)$ and a measurable function $H: S^{1} \times \cdots \times S^{1} \rightarrow \mathrm{C}$ by

$$
H\left(\alpha_{1}, \ldots, \alpha_{m}\right)=\sum_{i=1}^{k} a_{i} h_{i}(x) \alpha_{1}^{P_{i, 1}} \cdots \alpha_{m}^{P_{i, m}} .
$$

$R$ is ergodic and

$$
H\left(R^{n}(1, \ldots, 1)\right)=\sum_{i=1}^{k} a_{i}\left(\beta_{1}^{P_{i, 1}} \cdots \beta_{m}^{P_{t, m}}\right)^{n} h_{i}(x)
$$

is in $S^{1}$ for every $n$ (since $|\phi(x)|=1$ a.e.), so we must have $H\left(S^{1} \times \cdots \times S^{1}\right) \subset S^{1}$. Consider the function $H_{1}: S^{1} \rightarrow S^{1}$ given by $H_{1}(\alpha)=H(\alpha, 1, \ldots, 1)$. Since $H_{1}$ is a polynomial that takes $S^{1}$ into $S^{1}$, it must be a monomial, that is, $k=1$. Then $\phi(x) \cdots \phi\left(T^{n-1} x\right)=a_{1} \lambda_{1}^{n} h_{1}(x)$ a.e., and in particular, when $n=1, \phi(x)=$ $a_{1} \lambda_{1} h_{1}(x)$ a.e. We also have

$$
\phi(x)=\lambda_{1} h_{1}(x) / h_{1}(T x) \text { a.e., }
$$

and putting these together gives

$$
a_{1}=1 / h_{1}(T x) \text { a.e. }
$$

Thus $h_{1}$ is constant and, therefore, $\phi$ is constant, as desired. 
For the converse, if $\phi$ is constant, say $\phi(x)=\lambda$ a.e., we see that

$$
f_{x, \phi, T}(z)=\sum_{n=0}^{\infty} \lambda^{n} z^{n}=\frac{1}{1-\lambda z}
$$

is rational.

Corollary 2. Suppose that $\phi(x) \neq 0$ a.e. and that $\int_{X} \log |\phi| d \mu=0$. If there exist $\lambda$ in $S^{1}$ and a measurable function $h: X \rightarrow \mathrm{C}$ such that

$$
\phi(x)=\lambda h(T x) / h(x) \text { a.e., }
$$

then $f_{x, \phi, T}$ represents a rational function for almost every $x$ if and only if $h$ is a finite sum of eigenfunctions of $T$. In this case, the poles of $f_{x, \phi, T}$ are all simple and occur at

$$
\frac{1}{\lambda \lambda_{1}}, \frac{1}{\lambda \lambda_{2}}, \ldots, \frac{1}{\lambda \lambda_{k}},
$$

where $\lambda_{1}, \lambda_{2}, \ldots, \lambda_{k}$ are the eigenvalues corresponding to these eigenfunctions.

PROOF. We apply Theorem 3 to

$$
\begin{aligned}
f_{x, \phi, T}(z) & =\sum_{n=0}^{\infty} \lambda \frac{h(T x)}{h(x)} \lambda \frac{h\left(T^{2} x\right)}{h(T x)} \cdots \lambda \frac{h\left(T^{n} x\right)}{h\left(T^{n-1} x\right)} z^{n} \\
& =\frac{1}{h(x)} \sum_{n=0}^{\infty} h\left(T^{n} x\right)(\lambda z)^{n} .
\end{aligned}
$$

Proposition. Suppose that $\phi(x) \neq 0$ a.e., that $\int_{X} \log |\phi| d \mu=0$, and that $\phi(x)$ assumes only countably many values. Then $f_{x, \phi, T}$ represents a rational function if and only if $\phi$ is a simple function that can be written as

$$
\phi(x)=\sum_{i=1}^{k} b_{i} \chi_{B_{i}}(x) \text { a.e., }
$$

where each $B_{i}$ is measurable, $T B_{i}=B_{i+1}$ for $i=1,2, \ldots, k-1$, and $T B_{k}=B_{1}$.

Proof. Suppose first that $f_{x, \phi, T}$ is rational for almost every $x$. We can write

$$
\phi(x)=\sum_{i=1}^{\infty} b_{i} \chi_{B_{i}} \text { a.e., }
$$

where $\left\{B_{1}, B_{2}, B_{3}, \ldots\right\}$ is a measurable partition of $X$. Now there are only countably many sets of the form

$$
B_{i_{0}} \cap T^{-1} B_{i_{1}} \cap T^{-2} B_{i_{2}} \cap \cdots \cap T^{-p+1} B_{i_{p-1}},
$$

so at least one such set must have positive measure. Almost every orbit under $T$ must then hit this set infinitely often; thus, for almost every $x$, there are infinitely many values of $n$ for which

$$
\phi\left(T^{n} x\right)=b_{i_{0}}, \phi\left(T^{n+1} x\right)=b_{i_{1}}, \ldots, \phi\left(T^{n+p-1} x\right)=b_{i_{p-1}} .
$$

We also have, since $f_{x, \phi . T}$ is rational, an equation of the form

$$
\begin{aligned}
a_{0}+a_{1} \phi\left(T^{n} x\right) & +a_{2} \phi\left(T^{n} x\right) \phi\left(T^{n+1} x\right)+\cdots \\
& +a_{p} \phi\left(T^{n} x\right) \cdots \phi\left(T^{n+p-1} x\right)=0 \quad \text { a.e. }
\end{aligned}
$$


This means that the value of $\phi$ at any point on the orbit of $x$ is determined by the values of $\phi$ at the $p-1$ preceding points. Therefore, the sequence of values $\ldots, \phi\left(T^{-1} x\right), \phi(x), \phi(T x), \phi\left(T^{2} x\right), \ldots$ must be periodic for almost every $x$, with every orbit having the same period and the same sequence of values. This means that $\phi$ is actually (up to a set of measure zero) a simple function, and we can write $\phi(x)$ in the desired form.

For the converse, if $\phi$ is of the described form, then we have $\phi(x) \phi(T x) \cdots$ $\phi\left(T^{k-1} x\right)-b_{1} b_{2} \cdots b_{k}=0$ a.e., which makes $f_{x, \phi, T}$ rational almost everywhere.

\section{REFERENCES}

1. Jon Aaronson, An ergodic theorem with large normalizing constants, Israel J. Math. 38 (1981), $182-188$.

2. J. M. Anderson, J. Clunie and Ch. Pommerenke, On Bloch functions and normal functions, J. Reine Angew. Math. 270 (1974), 12-37.

3. Hirotada Anzai, Ergodic skew product transformations on the torus, Osaka Math. J. 3 (1951), 83-99.

4. Alexandra Ionescu Tulcea, Analytic continuation of random series, J. Math. Mech. 9 (1960), $399-410$.

5. __ Random series and spectra of measure-preserving transformations, Ergodic Theory (Fred B. Wright, ed.), Academic Press, New York, 1963, pp. 273-292.

6. Judy D. Halchin, Analvtic properties of random power series, $\mathrm{Ph} . \mathrm{D}$. dissertation, Univ. of North Carolina at Chapel Hill, 1983.

7. R. E. A. C. Paley and A. Zygmund, On some series of functions (1), (2), (3), Proc. Cambridge Philos. Soc. 26 (1930), 337-357, 458-474; 28 (1932), 190-205.

8. S. Saks and A. Zygmund, Analytic functions, Elsevier, New York, 1971.

9. W. T. Sledd, Random series which are BMO or Bloch, Michigan Math. J. 28 (1981), 259-266.

10. Hugo Steinhaus, Über die Wahrscheinlichkeit dafür, dass der Konvergenzkreis einer Potenzreihe ihre natürliche Grenze ist, Math. Z. 31 (1929), 408-416.

11. Sur la probabilité de la convergence de séries, Studia Math. 2 (1930), 21-39.

Department of Mathematics, University of North Carolina, Chapel Hill, North Carolina 27514

Department of Mathematics and Computer Science, The Citadel, Charleston, South Carolina 29409

Department of Mathematics, University of North Carolina, Chapel Hill, North Carolina 27514 (Current address of Karl Petersen)

Current address (Judy Halchin): Department of Mathematics, Eastern Illinois University, Charleston, Illinois 61920 